\title{
BAYESIAN FEEDBACK DETECTION FOR ADAPTIVE TRANSMISSION SYSTEMS
}

\author{
Anthony E. Ekpenyong and Yih-Fang Huang \\ University of Notre Dame \\ Department of Electrical Engineering \\ Notre Dame, IN 46556
}

\begin{abstract}
This paper considers adaptive transmission systems with imperfect feedback channels. It is shown that if the average SNR for the forward channel is known to the transmitter, the feedback detection can be modeled as a classical multiple hypotheses testing problem. Though maximum a posteriori detection is optimal compared to maximum likelihood (in the sense of minimum probability of error) from a feedback communication link viewpoint, the overall effect on adaptive system performance shows that ML detection could be better in the high SNR range. The MAP scheme is generalized to Bayesian detection by defining a cost function matrix, which assigns (unequal) weights to the feedback decision error probabilities. The result is close to ideal bit error rate (BER) performance with only a small drop in spectral efficiency.
\end{abstract}

\section{INTRODUCTION}

It is generally assumed in closed loop adaptive transmission systems that the feedback channel is perfect. This is justified by allowing for low-rate error-control coding to ensure feedback reliability [1-3]. However, in wireless communications, feedback is subject to noise and fading, and the allocated bandwidth is typically small so as to maximize useful data throughput. In addition, even though power control may be used to eliminate the effect of fading on feedback information [4], this comes at the price of increased energy usage for a mobile user. It is thus worthwhile to investigate the impact of feedback channel imperfections when uncoded information is sent back to the transmitter.

This paper analyzes the impact of a Bayesian feedback detection strategy on the performance of an adaptive modulation system (AMS). Since the average signal-to-noise ratio (SNR) for a slowly fading channel is subject to large-scale fading, it may be assumed to be constant with respect to small-scale fading. Thus, it is realistic to assume that the average SNR can be reliably fed back to the transmitter, where it can be used to compute the AMS state probabilities, which are also the (unequal) feedback signal transmission probabilities. The feedback communication system can then be formulated as a classical multiple hypotheses testing problem [5], and one may presume that maximum likelihood (ML) feedback detection is sub-optimal to maximum a posteriori (MAP) or, more generally, Bayesian detection. While this is true from a one-way

This work was supported in part by the Indiana 21st Century Fund for Research and Technology under Contract 651-1077-01, the U.S. Dept. of the Army under Contract DAAD 16-02-C-0057-P1, the National Science Foundation under Grant EC02-03366 and the Center for Applied Mathematics, University of Notre Dame. (feedback) communication link viewpoint, we show that the same cannot be said for the overall adaptive system performance.

Prior work in this area includes the adaptive trellis coded QAM system of [6], where the feedback information is protected by an error control code. The effect of arbitrary bit errors on a two-state system was shown in [4], where feedback power control was used to ensure a constant received power at the transmitter. The feedback scheme in [7] mitigates performance degradation due to feedback errors by employing a Finite State Markov Channel model. In this paper, we generalize the work of [4] to an $N$-state $(N>2)$ AMS without feedback power control, and compare ML and MAP feedback detection strategies. It is shown that the AMS BER performance for MAP feedback detection satisfies a target BER constraint over a practical SNR range, while the ML detection strategy fails at low SNR. Interestingly, it is seen that the AMS BER performance for ML feedback detection is better at high SNR compared to MAP detection. This seeming contradiction motivates the work in this paper. The MAP scheme can be generalized to Bayesian detection by defining a cost function matrix, which assigns (unequal) weights to the feedback decision error probabilities. The result is close to ideal bit error rate (BER) performance with only a small drop in spectral efficiency

Section 2 describes the adaptive system including the feedback communication model, while the feedback strategy is presented in Section 3. Performance results are shown in Section 4 and conclusions are given in Section 5.

\section{SYSTEM MODEL}

Consider an adaptive modulation system with $N$ QAM constellations, $\mathcal{M}=\left\{M_{i}\right\}_{i=0}^{N-1}$, operating over a flat fading Rayleigh channel. To focus on the effect of the feedback channel's imperfections, perfect channel knowledge at the receiver and negligible feedback delay are assumed. The SNR range for the forward channel can be partitioned as

$$
s_{i} \triangleq\left\{\gamma: \gamma_{i} \leq \gamma<\gamma_{i+1}\right\} \quad i=0,1, \ldots, N-1
$$

where $s_{0}$ is the outage state, $\gamma$ is the received SNR, and the region boundaries, $\left\{\gamma_{i}\right\}$, can be computed from approximate instantaneous BER expressions for $\mathcal{M}[1,3]$. If the feedback channel is perfect, $M_{i}$ is transmitted at the $n$th time instant only if $\gamma(n) \in s_{i}$. Now for a realistic feedback channel, the feedback modulator at the receiver is given by

$$
\phi\left(s_{i}\right) \rightarrow \mathcal{U}=\left\{\mathbf{u}_{0}, \mathbf{u}_{1}, \ldots, \mathbf{u}_{N-1}\right\}, \mathbf{u}_{i} \in\{-1,1\}^{b}
$$

where $\mathcal{U}$ is a set of vectors of BPSK symbols. Here, BPSK is chosen for its comparatively high noise immunity for uncoded transmission. The average energy of each symbol is set to unity, while 
$b=\left\lceil\log _{2} N\right\rceil$ is the number of bits required to uniquely describe $\mathcal{M}$. At the transmitter, the received feedback vector at the $n$th signaling instant is

$$
\mathbf{v}(n)=h^{\mathrm{fb}}(n) \mathbf{u}(n)+\mathbf{z}^{\mathrm{fb}}(n)
$$

where $h^{\mathrm{fb}} \sim \mathcal{C N}(0,1)$ is the feedback channel realization, and is assumed to be constant for $b$ symbols and known at the transmitter. The additive noise vector $\mathbf{z}^{\mathrm{fb}} \in \mathbb{C}^{b}$ consists of zero-mean independent and identically distributed (i.i.d.) elements having variance $\sigma_{\mathrm{fb}}^{2}$. For a feedback transmit power denoted as $P^{\mathrm{fb}}$, the feedback SNR per symbol is expressed as $\alpha=\left|h^{\mathrm{fb}}\right|^{2} P^{\mathrm{fb}} / \sigma_{\mathrm{fb}}^{2}$ with average SNR $\bar{\alpha}=P^{\mathrm{fb}} / \sigma_{\mathrm{fb}}^{2}$. The selection of $P^{\mathrm{fb}}$ is based on a designer-specified feedback channel quality. For example, for an AWGN feedback channel and a specified BER ${ }^{\mathrm{fb}}$, the instantaneous BER expression for ML BPSK detection may be inverted to give $P^{\mathrm{fb}}=\frac{1}{2}\left(Q^{-1} \mathrm{BER}^{\mathrm{fb}}\right)^{2}$, where $Q(\cdot)$ is the right-tail probability of the standard normal distribution and $\sigma_{\mathrm{fb}}^{2}=1$ without loss of generality. At the transmitter, the output of the feedback detector, $\hat{\mathbf{u}}=\psi(\mathbf{v})$, is used for rate adaptation.

Let $q_{i, j}$ denote the probability that $M_{i}$ is selected for transmission when $s_{j}$ is the true state. In this case, it can be shown that the transmission probability becomes $[4,7]$

$$
\pi_{i}^{\prime}=\sum_{j=0}^{N-1} q_{i, j} \pi_{j}
$$

where $\pi_{j}=\operatorname{Pr}\left(s_{j}\right)$ is the $j$ th state probability and is equivalent to the transmission probability in the absence of feedback errors. It can then be shown that the modified average spectral efficiency and BER are [7]

$$
\overline{\mathrm{SE}}=\sum_{i=1}^{N-1} \log _{2}\left(M_{i}\right) \pi_{i}^{\prime}
$$

and

$$
\begin{aligned}
\overline{\mathrm{BER}} & =(\overline{S E})^{-1} \sum_{i=1}^{N-1} \log _{2} M_{i} \\
& \times \sum_{j=0}^{N-1} q_{i, j} \int_{s_{j}} P_{b}\left(e \mid \gamma, M_{i}\right) p(\gamma) d \gamma
\end{aligned}
$$

where $P_{b}\left(e \mid \gamma, M_{i}\right)$ is the instantaneous BER expression for $M_{i}$ and $p(\gamma)$ is the probability density function (pdf) of $\gamma$. In this paper, system performance refers to the AMS.

The detection process $\psi(\cdot)$ determines the feedback decision error probability and, in turn, the system performance. In the next section we derive the decision error probability expressions for a Bayesian feedback detector.

\section{BAYESIAN FEEDBACK STRATEGY}

In most communication systems, transmitted symbols are assumed to be equally likely so that a minimum probability of error receiver is equivalent to an ML detector. However, this is not the case for the AMS feedback communication model considered here because feedback transmission is coupled to the instantaneous value of $\gamma$. Since $\pi_{i}$ is functionally dependent on the average SNR $\bar{\gamma}$ [1], the state probability vector $\pi=\left[\pi_{0}, \ldots, \pi_{N-1}\right]$ can be computed if $\bar{\gamma}$ is known at the transmitter. This implies that ML feedback detection may be sub-optimal to MAP, or more generally, Bayesian detection. The decision error probabilities in (3), (5) are then obtained by solving a classical multiple hypotheses testing problem, where the $i$ th hypothesis is defined as

$$
\mathcal{H}_{i}: \gamma \in s_{i}, \quad i=0,1, \ldots, N-1 .
$$

In the framework of detection theory, $q_{i, j} \triangleq \operatorname{Pr}\left(\mathcal{H}_{i} \mid \mathcal{H}_{j}\right)$ is the probability that the transmitter decides $\mathcal{H}_{i}$ when $\mathcal{H}_{j}$ is true. The optimum solution is obtained by minimizing the Bayes risk [5]

$$
\begin{aligned}
\bar{C} & =\sum_{i=0}^{N-1} \sum_{j=0}^{N-1} C_{i j} \operatorname{Pr}\left(\mathcal{H}_{i} \mid \mathcal{H}_{j}\right) \pi_{j} \\
& =\sum_{i=0}^{N-1} \int_{L_{i}} \bar{C}_{i}(\mathbf{v}) p(\mathbf{v}) d \mathbf{v}
\end{aligned}
$$

where $C_{i j}$ is the cost of choosing $\mathcal{H}_{i}$ when $\mathcal{H}_{j}$ is true, $p(\mathbf{v})$ is the pdf of $\mathbf{v}, L_{i}=\left\{\mathbf{v} \in \mathbb{C}^{b}: \mathcal{H}_{i}\right.$ is chosen $\}$ and

$$
\bar{C}_{i}(\mathbf{v})=\sum_{j=0}^{N-1} C_{i j} \operatorname{Pr}\left(\mathcal{H}_{j} \mid \mathbf{v}\right)
$$

Thus, the optimum detector chooses $\mathcal{H}_{i}$ if

$$
\bar{C}_{i}(\mathbf{v})<\bar{C}_{j}(\mathbf{v}) \quad \forall j \neq i .
$$

It is commonly assumed that $C_{i i}=0, C_{i j}=1 \forall i \neq j$. Hence, inserting these values in (8) and applying (9), it can be shown that the Bayes receiver is equivalent to a MAP detector, which selects $\mathcal{H}_{i}=\max _{j} \operatorname{Pr}\left(\mathcal{H}_{j} \mid \mathbf{v}\right)$ [5,8]. This is not necessarily the case for the feedback communication model considered here. For example, when $\gamma \in s_{0}$, i.e., the outage state, the cost of transmitting 64-QAM should be greater than the cost of transmitting BPSK since achieving the target BER constraint is of greater importance than maximizing the spectral efficiency.

The computation of $\operatorname{Pr}\left(\mathcal{H}_{i} \mid \mathcal{H}_{j}\right)$ for the multiple hypotheses problem is difficult because the decision regions, $\left\{L_{i}\right\}$, are determined by $N-1$-dimensional hyperplanes [5]. In Section 4, we present performance evaluation results obtained by simulation using (9). For the two-state system of [4], which becomes a binary hypotheses testing problem in this paper, closed form expressions can be obtained as shown in the next section.

\subsection{A two-state system}

Consider the binary hypothesis testing problem, $\left(\mathcal{H}_{0}, \mathcal{H}_{1}\right)$ and let $C_{j j}=0$ for any $j$. Then, (7) becomes

$$
\frac{p\left(\mathbf{v} \mid \mathcal{H}_{1}\right)}{p\left(\mathbf{v} \mid \mathcal{H}_{0}\right)} \underset{\mathcal{H}_{0}}{\stackrel{\mathcal{H}_{1}}{\gtrless}} \frac{C_{10} \pi_{0}}{C_{01} \pi_{1}}
$$

where $p\left(\mathbf{v} \mid \mathcal{H}_{j}\right)$ is equivalent to the multi-dimensional complex Gaussian pdf,

$$
p\left(\mathbf{v} \mid h^{\mathrm{fb}} \mathbf{u}_{j}\right)=\frac{1}{\left(\pi \sigma_{\mathrm{fb}}^{2}\right)^{b}} \exp \left(-\frac{1}{\sigma_{\mathrm{fb}}^{2}}\left\|\mathbf{v}-h^{\mathrm{fb}} \mathbf{u}_{j}\right\|_{2}^{2}\right) .
$$

Taking the logarithm of both sides of (10), the test statistic becomes

$$
\left\|\mathbf{v}-h^{\mathrm{fb}} \mathbf{u}_{0}\right\|^{2}-\left\|\mathbf{v}-h^{\mathrm{fb}} \mathbf{u}_{1}\right\|^{2} \underset{\mathcal{H}_{0}}{\stackrel{\mathcal{H}_{1}}{\gtrless}} \sigma_{\mathrm{fb}}^{2} \ln \frac{C_{10} \pi_{0}}{C_{01} \pi_{1}} .
$$


Defining $\eta=\ln \frac{C_{10} \pi_{0}}{C_{01} \pi_{1}}$ the test statistic can now be expressed as

$$
\begin{aligned}
D(\mathbf{v}) & \triangleq \operatorname{Re}\left\{\left(h^{\mathrm{fb}}\right)^{*}\left(\mathbf{u}_{1}-\mathbf{u}_{0}\right)^{T} \mathbf{v}\right\} \\
& -\frac{1}{2}\left|h^{\mathrm{fb}}\right|^{2}\left(\left\|\mathbf{u}_{1}\right\|^{2}-\left\|\mathbf{u}_{0}\right\|^{2}\right) \underset{\mathcal{H}_{0}}{\stackrel{\mathcal{H}_{1}}{\gtrless}} \frac{\sigma_{\mathrm{fb}}^{2}}{2} \eta,
\end{aligned}
$$

where $R e,{ }^{*},{ }^{T}$ denote, respectively, the real, conjugate and transpose operations. By noting that the transmitted sequences are real, it is straightforward to show that [8], conditioned on either hypothesis, $D(\mathbf{v})$ is Gaussian distributed with

$$
\begin{aligned}
& \mathrm{E}\left\{D \mid \mathcal{H}_{1}\right\}=1 / 2\left|h^{\mathrm{fb}}\right|^{2} d_{1,0}^{2} P^{\mathrm{fb}} \\
& \mathrm{E}\left\{D \mid \mathcal{H}_{0}\right\}=-1 / 2\left|h^{\mathrm{fb}}\right|^{2} d_{1,0}^{2} P^{\mathrm{fb}}
\end{aligned}
$$

and a common variance, $\sigma_{\mathrm{fb}}^{2} / 2\left|h^{\mathrm{fb}}\right|^{2} d_{1,0}^{2} P^{\mathrm{fb}}$, where $d_{1,0}$ is the Euclidean distance between $\mathbf{u}_{1}$ and $\mathbf{u}_{0}$. Therefore,

$$
\begin{aligned}
\operatorname{Pr}\left(\mathcal{H}_{0} \mid \mathcal{H}_{1}\right) & =\mathrm{E}_{\alpha}\left\{\operatorname{Pr}\left(D(\mathbf{v})<\frac{\sigma_{\mathrm{fb}}^{2}}{2} \eta \mid \mathcal{H}_{1}\right)\right\} \\
& =\mathrm{E}_{\alpha}\left\{Q\left(\sqrt{\frac{\left(d_{1,0}^{2} \alpha-\eta\right)^{2}}{2 d_{1,0}^{2} \alpha}}\right)\right\}
\end{aligned}
$$

Similarly, the expression for $\operatorname{Pr}\left(\mathcal{H}_{1} \mid \mathcal{H}_{0}\right)$ is obtained by changing the sign in the squared term of $Q(\cdot)$ in (15).

To analyze the performance of the system, let $C_{01}=C_{10}$. In general, $\pi_{0} \neq \pi_{1}$, resulting in a decision biased in favor of the more likely hypothesis i.e.,

$$
\begin{aligned}
\operatorname{Pr}\left(\mathcal{H}_{0} \mid \mathcal{H}_{1}\right) & <\operatorname{Pr}\left(\mathcal{H}_{1} \mid \mathcal{H}_{0}\right) \text { for } \eta<0 \\
& >\operatorname{Pr}\left(\mathcal{H}_{1} \mid \mathcal{H}_{0}\right) \text { for } \eta>0
\end{aligned}
$$

since $Q(\cdot)$ is a decreasing function of its argument.

At low SNR, $\pi_{0}>\pi_{1}$ so that the inequality $\operatorname{Pr}\left(\mathcal{H}_{1} \mid \mathcal{H}_{0}, \alpha\right)<$ $\operatorname{Pr}\left(\mathcal{H}_{0} \mid \mathcal{H}_{1}, \alpha\right)$ holds. This is desirable from the AMS perspective because a wrong decision in favor of $\mathcal{H}_{1}$ increases the BER. Conversely, $\operatorname{Pr}\left(\mathcal{H}_{1} \mid \mathcal{H}_{0}, \alpha\right)>\operatorname{Pr}\left(\mathcal{H}_{0} \mid \mathcal{H}_{1}, \alpha\right)$ at higher SNR. Therefore, if $\operatorname{Pr}\left(\mathcal{H}_{1} \mid \mathcal{H}_{0}, \alpha\right)$ is greater for MAP compared to ML detection, the BER would correspondingly be higher because the decision favors $\mathcal{H}_{1}$, implying more frequent transmission. It should be noted that the average BER of the feedback channel with MAP detection is, in general, better than ML detection. However, for the coupled system, the individual crossover probabilities are of more significance to the AMS $\overline{\mathrm{BER}}$ performance. This clearly indicates that the well-known sub-optimality of ML to MAP detection, in terms of BER performance, is strictly based on a one-way communication link perspective. The overall effect of feedback error on the adaptive system performance would show that, at high SNR, the $\overline{\mathrm{BER}}$ for MAP is higher than that for ML feedback detection. This effect is similarly present for the more general AMS when $N>2$ as shown in Section 4.

\subsection{Bayesian Cost Function}

While equal cost functions are the norm in traditional communication systems, the importance of the $\overline{\mathrm{BER}}$ being as small as possible, and not simply to satisfy the $\mathrm{BER}_{T}$, may supersede the desire for maximal spectral efficiency in some applications. We can then generalize MAP to Bayesian feedback detection by defining suitable cost functions.
To define a cost function matrix for minimum average BER, it is desirable that $C_{i j}>C_{j i}$ and $C_{i j}>C_{k j}$ for $i>k>j$. It is difficult to quantitatively assign costs in general due to the subjective nature of the impact of wrong decisions on a system. In this paper, we use the cost function

$$
C_{i j}=|i-j|, C_{j i}=\beta C_{i j} \quad i>j, \beta \in(0,1] .
$$

In other words, the cost of transmitting at a higher rate than what the channel state calls for, is higher than the cost of transmitting conservatively. It would be shown that the choice of $\beta$ influences the system performance.

\section{RESULTS}

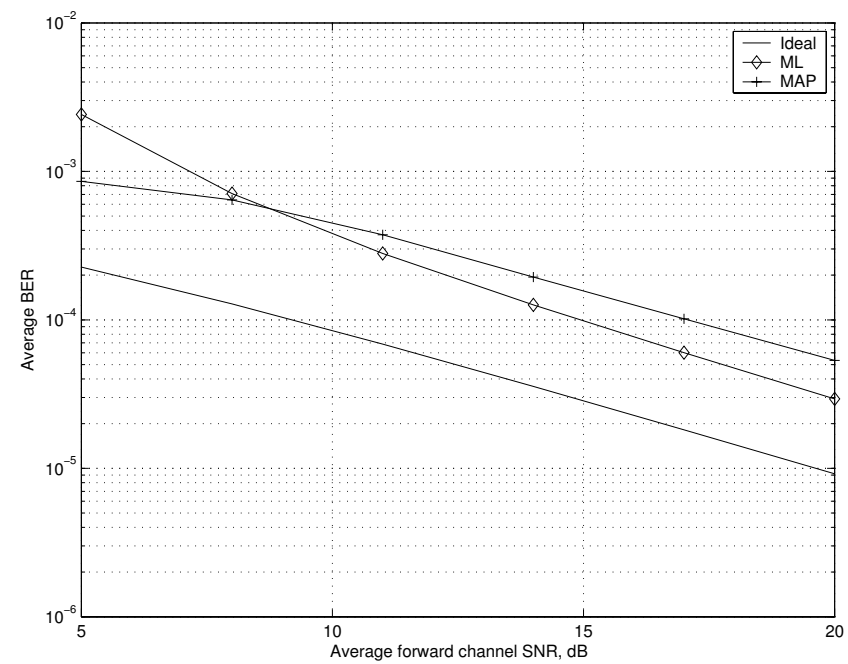

Fig. 1. Average BER for an $N=2$ AMS using ML, MAP detection for a fading feedback channel.

For all results in this section $\mathrm{BER}_{T}=10^{-3}$. The analytical performance of a two-state system for an outage state and BPSK modulation is shown in Fig. 1 for a fading feedback channel with $\bar{\alpha}=15 \mathrm{~dB}$. It can be seen that at low SNR, ML detection fails to satisfy the $\mathrm{BER}_{T}$ while the BER curve for the MAP scheme is lower than the $\mathrm{BER}_{T}$ over the SNR range. However, there is a crossover point at $\bar{\gamma}=8.75 \mathrm{~dB}$ after which the ML gives better performance. This validates our earlier analysis of the impact of these detection strategies.

An adaptive M-QAM system is simulated with 7 non-zero QAM constellations, $\left\{2^{i}-\mathrm{QAM}\right\}_{i=1}^{7}$ over a flat fading Rayleigh channel with a normalized Doppler frequency of $10^{-3}$. Fig. 2 shows the average BER performance for an AWGN feedback channel where $P^{\mathrm{fb}}$ is obtained by setting $\mathrm{BER}_{\mathrm{fb}}=10^{-3}$ and $\sigma_{\mathrm{fb}}^{2}=1$ for ML detection. The MAP/Bayesian schemes are implemented using (7). A similar trend for the two-state system is also observed with a crossover point at $\bar{\gamma} \approx 20.5 \mathrm{~dB}$. There are only negligible differences in the spectral efficiency and thus, it is not shown here. It should be noted, though, that from the point of view of satisfying the $\mathrm{BER}_{T}$, MAP is optimum over the whole SNR range. Using the cost function of (17) with $\beta=0.01$, the performance is close to the ideal case. However, there is a small penalty in the spectral efficiency of less than $0.02 \mathrm{bps} / \mathrm{Hz}$ at high SNR. 


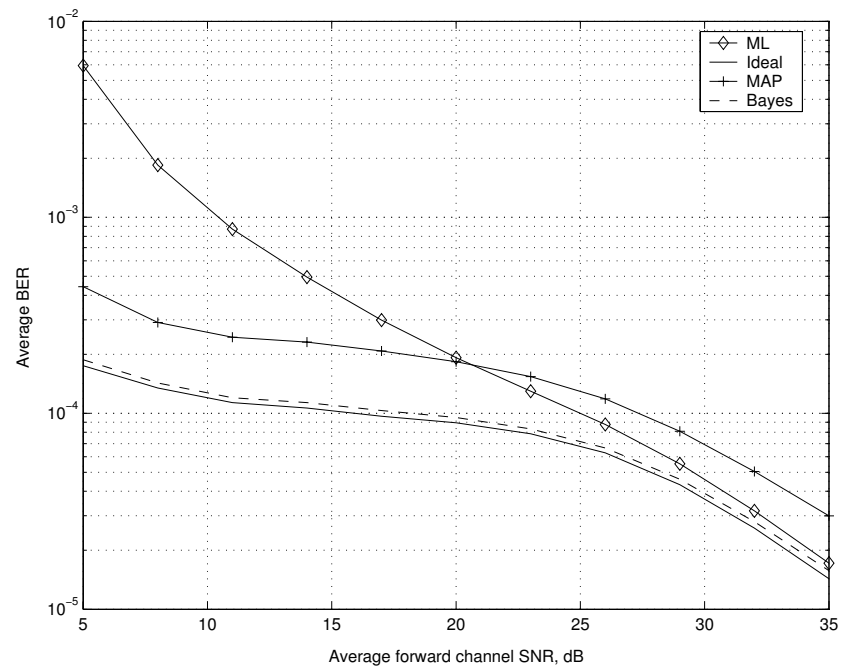

Fig. 2. Average BER for an $N=8$ AMS using ML, MAP and Bayesian detection over an AWGN feedback channel.

The $\overline{\mathrm{BER}}$ performance difference is even more significant for a fading feedback channel as shown in Fig. 3. The average SNR of the feedback link is set to $\bar{\alpha}=15 \mathrm{~dB}$. The ML detector cannot be used for $\bar{\gamma}<19.5 \mathrm{~dB}$, while the MAP can be used over the full SNR range. Again in the high SNR region the ML gives improved performance compared to MAP. For the Bayesian schemes shown in Fig. 3, the effect of varying $\beta$ results in the $\overline{\mathrm{BER}}$ performance approaching that of the ideal feedback channel but there is a small loss in spectral efficiency of $0.03,0.13 \mathrm{bps} / \mathrm{Hz}$ respectively for $\beta=0.1,0.01$. Therefore, there is a trade-off between lowering the $\overline{\mathrm{BER}}$ as much as possible and maximizing the spectral efficiency.

\section{CONCLUSION}

It is shown that if the average SNR for the forward channel is known to the transmitter, MAP feedback detection consistently results in an AMS average BER performance lower than the target BER constraint while ML feedback detection does not. However, an interesting phenomenon is shown where ML may outperform MAP in the high SNR region. For applications where a small decrease in spectral efficiency can be traded for lower BER, a Bayesian cost may be defined to achieve close to ideal BER performance for the AMS. The main assumption used in the paper, namely that the average SNR is known to the transmitter, could be relaxed by formulating the problem as a minimax multiple hypotheses problem. This would be pursued in future work.

\section{REFERENCES}

[1] M. S. Alouini and A. J. Goldsmith, "Adaptive Modulation over Nakagami Fading Channels," Wireless Personal Communications, vol. 13, no. 1, pp. 119-143, May 2000.

[2] D. L. Goeckel, "Adaptive coding for time-varying channels using outdated fading estimates," IEEE Trans. Commun., vol. 47, no. 6, pp. 844-855, June 1999.

[3] S. Zhou and G. B. Giannakis, "How Accurate Channel Prediction needs to be for Transmit-Beamforming with Adaptive
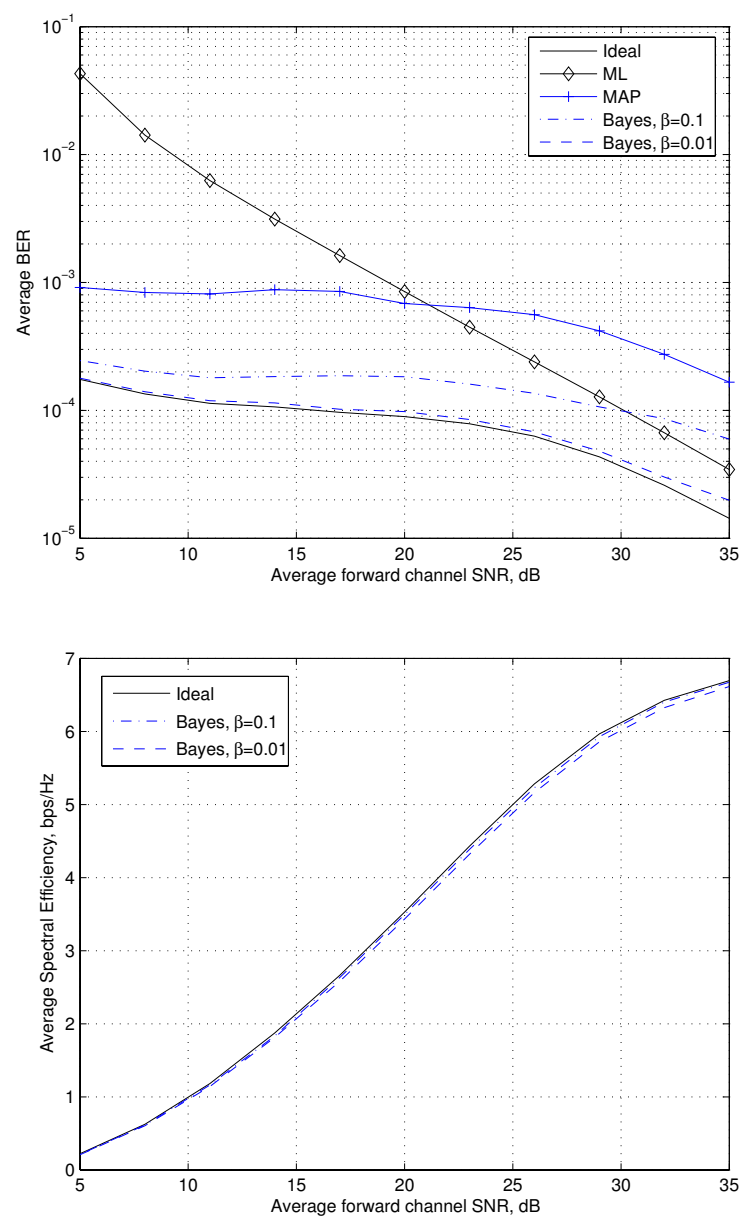

Fig. 3. Average BER and SE for an $N=8$ AMS using ML, MAP and Bayesian detection over a fading feedback channel with $\bar{\alpha}=15$ $\mathrm{dB}$.

Modulation over Rayleigh MIMO Channels," in Proceedings of Int. Conf. on Acoustics, Speech and Signal Processing, Hong Kong, 2003.

[4] J. Hämäläinen and R. Wichman, "Bit Error Probabilities in a Two-Rate Communication System," in Proceedings of IEEE Int. Conf. Commun., Anchorage, Alaska, 2003.

[5] H. L. Van Trees, Detection, Estimation, and Modulation Theory, Part 1, John Wiley \& Sons, New York, 1968.

[6] V. K. N. Lau and M. D. Macleod, "Variable-rate adaptive trellis coded QAM for flat-fading channels," IEEE Trans. Commun., vol. 49, no. 9, pp. 1550-1560, Sept. 2001.

[7] A. E. Ekpenyong and Y-F. Huang, "Markov Channel-Based Feedback Schemes for Adaptive Modulation Systems," in Proc. IEEE Globecom, Dallas, TX, 2004.

[8] S. M. Kay, Fundamentals of Statistical Signal Processing, Volume 2 - Detection Theory, Prentice-Hall, Englewood Cliffs, NJ, 1998. 